\title{
COVID-19 infection and beta thalassemia; a single center experience in Iran
}

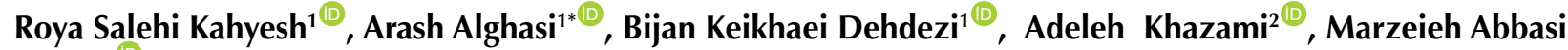 \\ Nasab $^{1}$
}

${ }^{1}$ Thalassemia and Hemoglobinopathies Research centre, research institute of Health, Ahvaz Jundishapur University of Medical Sciences, Ahvaz, Iran

${ }^{2}$ Baqaei2 hospital, Ahvaz Jundishapur University of Medical Sciences, Ahvaz, Iran

\section{Correspondence to: \\ Arash Alghasi , Email; arashalqasi@yahoo.com, \\ alghasi-a@ajums.ac.ir}

Received: 20 Apr. 2021 Accepted: 1 June 2021 ePublished: 10 June 2021

Keywords: Hemoglobinopathy, COVID-19, Beta thalassemia

\begin{abstract}
Introduction: COVID-19 infection is a contagious acute respiratory syndrome, leading to a high mortality rate; it is also a systemic disease, and all people are at risk of being infected, including hemoglobinopathies patients. Thalassemia is one of the most prevalent hemoglobinopathies while Beta-thalassemia patients, are at risk for the deadly effects of the coronavirus.

Objectives: This study examines the prevalence, severity, and mortality rate of COVID-19 infection in thalassemia patients of the southwest of Iran.

Patients and Methods: In this descriptive cross-sectional study, from March 2019 to September 2020, 1000 beta-thalassemia patients, referred to Shahid Baqaei 2 hospital, Ahvaz, Iran, were screened for COVID-19. Results: Findings showwd $80 \%$ of thalassemia patients were women. Fever, body aches, weakness, and dizziness were the main symptoms of patients.

Conclusion: This result suggests that hemoglobinopathies may affect infections and mortality from COVID-19 infection. However, our findings do not support a direct association between hemoglobin disease and COVID-19, and further research is needed to confirm this, but this might state the hemoglobin's role in the pathophysiology of COVID-19 infection.
\end{abstract}

\section{Citation: Salehi Kahyesh R, Alqasi A, Keikhaei Dehdezi B, Khazami A, Abbasi Nasab M. COVID-19 infection and beta thalassemia; a single center experience in Iran. J Prev Epidemiol. 2021;6(2):e24. doi: 10.34172/jpe.2021.24}

\section{Introduction}

Around a month after the first diagnosis of severe acute respiratory syndrome-related coronavirus (SARS-CoV-2), on January 31, 2020, the World Health Organization (WHO) announced that SARS-CoV-2 is becoming a severe health threat to humans. Unfortunately, in a short time, it was turned into a deadly pandemic disease (1). The SARS-CoV-2 (COVID-19) has the positive-strand RNA, which encoded structural and non-structural proteins (ORF8, ORF7a, ORF6, ORF3a, ORF10, and orf1ab). The primary structural proteins include the envelope protein (E), spike surface glycoprotein (S), nucleocapsid phosphoprotein, and matrix protein (M) (2). The angiotensin-converting enzyme 2 (ACE2) protein is the central COVID-19 receptor in the host, binds to the spike protein (3), and identified in various human organs, including the respiratory system, thymus, bone marrow, gastrointestinal tract, liver, spleen, kidney, lymph nodes and brain (4). Therefore, it can cause infections in various systems of the human body

\section{Key point}

The result of our study showed that hemoglobinopathies might affect COVID-19 mortality.

(5). The main presentation of infection includes fatigue, dry cough, dizziness, fever above $37.3^{\circ} \mathrm{C}$, sore throat, headache, myalgia, difficulty breathing or shortness of breath, hemoptysis, and gastrointestinal symptoms $(6,7)$. Examination of biochemical parameters performed on some patients with novel coronavirus-induced pneumonia revealed an abnormal phenomenon associated with hemoglobin biochemical indexes (8). These studies have shown that COVID-19 patients' hemoglobin is declined $(5,9)$. As the COVID-19 virus needs many porphyrins, it is thought that some specific COVID-19 proteins (ORF8, ORF10, and ORF3a) could coordinately attack red blood cells (RBCs) and lead to its hemolysis. The heme molecule is a vital component of RBC hemoglobin, composed of porphyrin and iron. COVID-19 proteins could capture heme and dissociate the porphyrin and

Copyright $\odot 2021$ The Author(s); Published by Society of Diabetic Nephropathy Prevention. This is an open-access article distributed under the terms of the Creative Commons Attribution License (http://creativecommons.org/licenses/by/4.0), which permits unrestricted use, distribution, and reproduction in any medium, provided the original work is properly cited. 
release the iron ions (10). On one hand, a decrease in hemoglobin concentration in COVID-19 patients leads to decreased oxygen delivery to the tissues involved in the infection. It can lead to septic shock or multiple organ dysfunction syndromes, and on the other hand, the body has faced over-accumulating harmful iron ions that cause inflammation. Thereby, the cells producing large amounts of ferritin to bind to free iron ions and reduce damage (11, 12). People with diabetes have extreme inflammation due to excess iron, carbon dioxide, and oxygen. Patients with respiratory distress will worsen, and organs and tissues of the whole body encounter varying degrees of damage (10).

$\beta$-thalassemia is the most common monogenic recessive disease worldwide caused by decreased or complete absent synthesis of $\beta$-globin chains (responsible for hemoglobin production in the RBC) (13). Besides, the immune system of thalassemia patients is altered. It is associated with the decreased neutrophil count, changes in the number and function of natural killer cells, macrophage dysfunction and phagocytosis, and the production of interferon-gamma (IFN- $\gamma$ ) which can lead to hypersensitivity to infectious agents $(14,15)$. Followed by, some other comorbidities may make them vulnerable to the virus, including liver disease, diabetes, and severe iron overload (16). Thus, thalassemia patients are expected to be more exposed to severe symptoms of the COVID-19 $(19,21)$. Iran is on the thalassemia world belt map with around two million $\beta$-thalassemia carriers and $25000 \beta$ thalassemia major patients. Khuzestan province is located in southwest Iran, and the frequency of $\beta$-thalassemia is too high due to the existence of different ethnicities (Arab, Bakhtiari, Qashqai, Persian, and Lor) and consanguineous marriages (17).

\section{Objectives}

This study investigates the prevalence, severity, and mortality rate of COVID-19 in thalassemia patients referred to Baqaei 2 hospital in Iran's southwest.

\section{Patients and Methods}

\section{Study design}

Baqaei 2 hospital is a referral center for patients with hemoglobin disorders in Khuzestan province. The betathalassemia patients referred to this center were screened for COVID-19 from March 2019 to September 2020. To this end, some leading indicators were evaluated, such as their lung CT scan, CBC, C-reactive protein (CRP); lactate dehydrogenase (LDH); creatine phosphokinase (CPK). tests, and blood oxygen levels.

\section{Statistical analysis}

Data were analyzed using SPSS software version 22. MannWhitney U, chi-square and Pearson's correlation tests were applied. Data were expressed as mean \pm standard deviation. $P<0.05$ was considered statistically significant.

\section{Results}

Out of $1000 \beta$-thalassemia patients referred to Baqaei 2 hospital, 10 patients were positive for COVID-19. Eighty percent of thalassemia patients infected were women, and their age range was from 10 to 49 years. Fever, body aches, weakness, and dizziness were the main symptoms of patients, and in terms of laboratory indexes, increased WBC count and ESR and decreased platelets were observed in most patients. Fortunately, no mortality was observed among them (Table 1).

\section{Discussion}

In a similar study, Karimi et al have evaluated the COVID-19 severity among $48 \beta$-thalassemia patients and observed that $73.9 \%$ had mild to moderate symptoms and recovered; however $26.1 \%$ of their patients died. More than $60.0 \%$ of patients had at least one comorbidity in this study. Diabetes, hypertension, pulmonary artery hypertension, and heart disease in dead patients were significantly higher. According to their reports, the prevalence of COVID-19 in thalassemia patients is 8.17 per 10000 versus 11.01 per 10000 in the general population (18). Some studies have focused on the hemoglobin targeting by SARS-CoV-2 and its mechanism of action. They have shown that it may attack hemoglobin, target the heme, and acquire porphyrins so it is believed that $\beta$-thalassemia might protect patients against COVID-19 $(10,19)$. Liu et al found that the orflab, ORF3a, and ORF10 viral proteins have participated in attacks to heme. Through analysis, they found that in oxidized hemoglobin, the orf1ab (His300) first attacked the alpha chain, and then the ORF3a (Gln303) and ORF10 successively attacked the beta chain, and at last IIe304 connects to the iron heme. This state leads to a decrease in hemoglobin levels and, consequently, a decrease in oxygen supply. For de-oxyhemoglobin, the possible mechanism is that orflab first attacks the 1-beta chain, and then ORF3a and ORF10 directly docked to the heme of the beta chain and dissociated it, therefore increasingly decreased hemoglobin that can carry carbon dioxide and blood sugar. These results show that the higher hemoglobin content could be associated with a higher risk of disease (20). In this regard, Lansiaux et al, suggested that the heterozygous ß-thalassemia patients may develop immunity to SARSCoV-2 viral infection (using multiple linear regression analysis) (21). Another study was conducted in 17 centers in 10 countries, and the results confirmed our data (22). In this study, 9499 patients with hemoglobinopathies ( $\beta$-thalassemia and sickle cell disease) were enrolled and identified only 13 patients with COVID-19 with clinical manifestations ranging from asymptomatic to severe pneumonia and respiratory failure (22). However, there is no strong clinical evidence and validated studies to support that hemoglobinopathies patients are less at risk for the relatively severe form of COVID-19, and achieving this result requires further research in this area. Therefore, 
Table 1. Information of thalassemia patients with COVID-19

\begin{tabular}{|c|c|c|c|c|c|}
\hline No. & Gender & Age & Type of disease & Sign & Lab. Index \\
\hline 1 & $\mathrm{~F}$ & 34 & Thalassemia intermediate & Weakness and lethargy & ESR $\uparrow$, PLT.N, Increased blood bilirubin $\uparrow$ \\
\hline 2 & $\mathrm{~F}$ & 28 & Thalassemia intermediate & Cough, epigastric pain & $\mathrm{ESR} \uparrow, \mathrm{PLT} \uparrow$, lymph$\uparrow$ \\
\hline 3 & M & 10 & Thalassemia major & $\begin{array}{l}\text { Fever, body pains, weakness and lethargy, cough, } \\
\text { fatigue }\end{array}$ & ESR $\uparrow$, Hypoglycemia $\downarrow$, PLT.N \\
\hline 4 & M & 20 & Thalassemia major & Fever, abdominal pain, diarrheal, & PLT $\downarrow$ \\
\hline 5 & $\mathrm{~F}$ & 28 & Thalassemia major & Body pain, high fever & PLT.N, Blood in the urine lymph $\downarrow, \mathrm{ESR} \uparrow, \mathrm{FBS} \uparrow$ \\
\hline 6 & $\mathrm{~F}$ & 27 & Thalassemia major & Weakness and lethargy & $\mathrm{WBC} \uparrow$ \\
\hline 7 & $\mathrm{~F}$ & 39 & Thalassemia major & Fever, cough & $\mathrm{WBC} \uparrow$ \\
\hline 8 & $\mathrm{~F}$ & 29 & Thalassemia major & Headache, body pains, nausea, vomiting & Increase CRP, PLt.WBC $\uparrow$ \\
\hline 9 & $\mathrm{~F}$ & 8 & Thalassemia major & Fever, headache, unconsciousness & $\mathrm{PLT} \downarrow, \mathrm{ESR} \uparrow$ \\
\hline 10 & $\mathrm{~F}$ & 49 & Thalassemia major & Fever, chills & PLT. $\uparrow, W B C \uparrow$ \\
\hline
\end{tabular}

thalassemia patients should be taken care of with extra caution.

\section{Conclusion}

According to the results of several studies, it can be concluded that hemoglobinopathies may affect COVID-19 mortality. Of course, various studies have displayed inconsistent results, which indicates the need for further investigations in this regard. Although our finding does not prove the direct association between hemoglobin structure and COVID-19 mortality definitively, we suggest evaluation of hemoglobin's role in the pathophysiology of COVID-19.

\section{Authors' contribution}

AA, RSK and BKD were the principal investigators of the study. $A K$ and MAN were included in preparing the concept and design. AA and RSK revised the manuscript and critically evaluated the intellectual contents. All authors participated in preparing the final draft of the manuscript, revised the manuscript and critically evaluated the intellectual contents. All authors have read and approved the content of the manuscript and confirmed the accuracy or integrity of any part of the work.

Conflicts of interest

The authors declare that they have no competing interests.

\section{Ethical issues}

The research followed the tenets of the Declaration of Helsinki. The Ethics Committee of Ahvaz Jundishapur University of Medical Sciences approved this study. The institutional ethical committee at Ahvaz Jundishapur University of Medical Sciences approved all study protocols (IR.AJUMS.REC.1399.723). Accordingly, written informed consent was taken from all participants before any intervention. This study was extracted from M.D., thesis of (Grant \# Th-9913 (at this university. Moreover, ethical issues (including plagiarism, data fabrication, double publication) have been completely observed by the authors.

\section{Funding/Support}

This research has been done with the support of Ahvaz Jundishapur University.

\section{References}

1. Jan $\mathrm{H}$, Faisal $\mathrm{S}$, Khan $\mathrm{A}$, Khan $\mathrm{S}$, Usman $\mathrm{H}$, Liaqat $\mathrm{R}$, et al. COVID-19: Review of Epidemiology and Potential Treatments
Against 2019 Novel Coronavirus. Discoveries (Craiova). 2020 26;8:e108. doi: 10.15190/d.2020.5.

2. Wu A, Peng Y, Huang B, Ding X, Wang X, Niu P, et al. Genome Composition and Divergence of the Novel Coronavirus (2019. nCoV) Originating in China. Cell Host Microbe. 2020;27:325328. doi: 10.1016/j.chom.2020.02.001.

3. Li F. Structure, Function, and Evolution of Coronavirus Spike Proteins. Annu Rev Virol. 2016:3:237-261. doi: 10.1146/ annurev-virology-110615-042301.

4. Hamming I, Timens W, Bulthuis ML, Lely AT, Navis G, van Goor $\mathrm{H}$. Tissue distribution of ACE2 protein, the functional receptor for SARS coronavirus. A first step in understanding SARS pathogenesis. J Pathol. 2004;203(2):631-7. doi: 10.1002/ path. 1570.

5. Espinosa OA, Zanetti ADS, Antunes EF, Longhi FG, Matos TA, Battaglini PF. Prevalence of comorbidities in patients and mortality cases affected by SARS-CoV2: a systematic review and meta-analysis. Rev Inst Med Trop Sao Paulo. 2020;62:e43. doi: 10.1590/S1678-9946202062043.

6. Huang C, Wang Y, Li X, Ren L, Zhao J, Hu Y, et al. Clinical features of patients infected with 2019 novel coronavirus in Wuhan, China. Lancet. 2020;395:497-506. doi: 10.1016/ S0140-6736(20)30183-5.

7. She J, Liu L, Liu W. COVID-19 epidemic: Disease characteristics in children. J Med Virol. 2020;92:747-54. doi: 10.1002/ jmv. 25807.

8. Chen N, Zhou M, Dong X, Qu J, Gong F, Han Y, et al. Epidemiological and clinical characteristics of 99 cases of 2019 novel coronavirus pneumonia in Wuhan, China: a descriptive study. Lancet. 2020;395:507-513. doi: 10.1016/ S0140-6736(20)30211-7.

9. Taneri PE, Gómez-Ochoa SA, Llanaj E, Raguindin PF, Rojas LZ, Roa-Díaz ZM, et al. Anemia and iron metabolism in COVID-19: a systematic review and meta-analysis. Eur J Epidemiol. 2020;35:763-73. doi: 10.1007/s10654-02000678-5.

10. Liu W, Li H. COVID-19 disease: ORF8 and surface glycoprotein inhibit heme metabolism by binding to porphyrin. ChemRxiv. 2020. doi: 10.26434/chemrxiv.11938173

11. San Juan I, Bruzzone $C$, Bizkarguenaga $M$, Bernardo-Seisdedos G, Laín A, Gil-Redondo R, et al. Abnormal concentration of porphyrins in serum from COVID-19 patients. Br J Haematol. 2020;190:e265-7. doi: 10.1111/bjh.17060.

12. Pan F, Ye T, Sun P, Gui S, Liang B, Li L, et al. Time Course of Lung Changes at Chest CT during Recovery from Coronavirus Disease 2019 (COVID-19). Radiology. 2020;295:715-721. doi: 10.1148/radiol.2020200370.

13. Zhou F, Yu T, Du R, Fan G, Liu Y, Liu Z, et al. Clinical course and risk factors for mortality of adult inpatients with COVID-19 
in Wuhan, China: a retrospective cohort study. Lancet. 2020;395:1054-1062. doi: 10.1016/S0140-6736(20)30566-3.

14. Xu XM, Zhou YQ, Luo GX, Liao C, Zhou M, Chen PY, et al. The prevalence and spectrum of alpha and beta thalassaemia in Guangdong Province: implications for the future health burden and population screening. J Clin Pathol. 2004;57:51722. doi: 10.1136/jcp.2003.014456.

15. Kosaryan M, Zafari M, Alipur A, Hedayatizadeh-Omran A. The effect and side effect of hydroxyurea therapy on patients with $\beta$-thalassemia: a systematic review to December 2012. Hemoglobin. 2014;38:262-71. doi: 10.3109/03630269.2014.927770.

16. Derchi G, Galanello R, Bina P, Cappellini MD, Piga A, Lai ME, et al. Webthal Pulmonary Arterial Hypertension Group. Prevalence and risk factors for pulmonary arterial hypertension in a large group of $\beta$-thalassemia patients using right heart catheterization: a Webthal study. Circulation. 2014;129:33845. doi: 10.1161/CIRCULATIONAHA.113.

17. Nakavachara P, Viprakasit V. Adrenal insufficiency is prevalent in $\mathrm{HbE} / \beta$-thalassaemia paediatric patients irrespective of their clinical severity and transfusion requirement. Clin Endocrinol (Oxf). 2013;79:776-83. doi: 10.1111/cen.12235.
18. Taher AT, Bou-Fakhredin R, Kreidieh F, Motta I, De Franceschi L, Cappellini MD. Care of patients with hemoglobin disorders during the COVID-19 pandemic: An overview of recommendations. Am J Hematol. 2020;95:E208-10. doi: 10.1002/ajh.25857.

19. Nezhad FH, Nezhad KH, Choghakabodi PM, Keikhaei B. Prevalence and Genetic Analysis of $\alpha$ - and $\beta$-Thalassemia and Sickle Cell Anemia in Southwest Iran. J Epidemiol Glob Health. 2018;8:189-95. doi: 10.2991/j.jegh.2018.04.103.

20. Karimi M, Haghpanah S, Azarkeivan A, Matin S, Safaei A, De Sanctis V. Coronavirus Disease 2019 (COVID-19) Severity in Patients with Thalassemias: A Nationwide Iranian Experience. Mediterr J Hematol Infect Dis. 2021;13:e2021008. doi: 10.4084/MJHID.2021.008.

21. Lansiaux E, Pébaÿ PP, Picard JL, Son-Forget J. COVID-19: beta-thalassemia subjects immunised? Med Hypotheses. 2020;142:109827. doi: 10.1016/j.mehy.2020.109827.

22. Drouin E. Beta-thalassemia may protect against COVID 19. Med Hypotheses. 2020;143:110014. doi: 10.1016/j. mehy.2020.110014. 